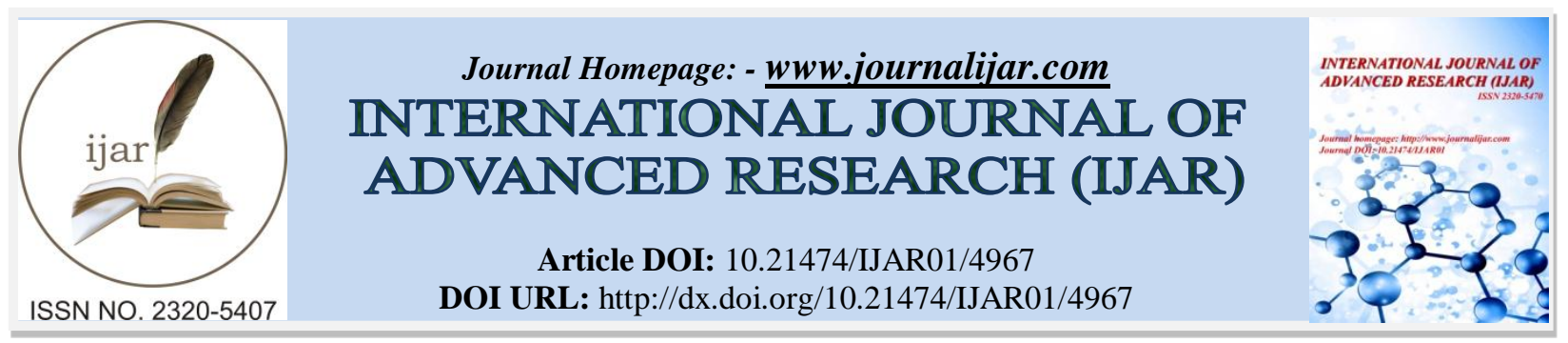

RESEARCH ARTICLE

\title{
RELIABILITY OF FINITE MIXTURE OF COMPONENTS.
}

N. Swathi.

Dept. of Mathematics, Kakatiya University, Warangal, Telangana State-506009.

\section{Manuscript Info}

Manuscript History

Received: 26 May 2017

Final Accepted: 28 June 2017

Published: July 2017

Key words:-

Time dependent stress-strength model,

Random- fixed, Random independent,

Finite mixture of exponential

Distribution, Reliability.

\section{Abstract}

Failure of a system may occur due to certain type of stresses acting on them. If these stresses do not exceed a certain threshold value the system may work for a long period. On the other hand, if the stresses exceed the threshold they may fail within no time. There is uncertainty about stress and strength random variables at any instant of time and also about the behavior of the variables with respect to time and cycles. Time dependent stress- strength models are considered with repeated application of stress and also the change of the strength with time. Reliability of time dependent stress- strength system is carried out by considering stress is random independent and strength is random- fixed. In this paper to find the reliability of finite mixture model where strength follow finite mixture of exponential distribution and stress follows exponential distribution.

Copy Right, IJAR, 2017,. All rights reserved.

\section{Introduction:-}

Reliability of a system is the probability that the system will adequately perform its intended purpose for a given period of time under stated environmental conditions. In some cases system failure occur due to certain type of stresses acting on them. Thus system composed of random strengths will have its strength as random variable and the stress applied on it will also be a random variable. A system fail whenever an applied stress exceeds strength of the system.

In reliability theory, there are lots of real life situation where the concept of mixture distributions can be applied. For example, in life testing experiments, the systems will be failed due to different causes and the time to failure due to different reasons are likely to follow different distributions. Knowledge of these distributions is essential to eliminate cause of failures and thereby to improve the reliability. Maya, T.Nair described the estimation of reliability based on finite mixture of pareto and beta distributions. Time dependent stress- strength models are considered with repeated application of stress and also the change of the strength with time. "Stress" is used to indicate any agency that tends to induce "failure", while "strength" indicates any agency that resisting "failure". "Failure" is defined to have occurred when the actual stress exceeds the actual strength.

There is Uncertainty about stress and strength random variables at any instant of time and also about the behavior of the variables with respect to time and cycles. These three terms, "deterministic", "random fixed" and "random independent" are used to describe these two uncertainties In deterministic the variables assume values that exactly known a priori, random fixed refers to the behavior of the variable with respect to time is fixed or the variables varies with time in a known manner, random independent means the successive stresses are generally independent, strength will vary randomly and will be independent from cycle to cycle. 
The reliability after $n$ cycles is referred to $R(t)$, the reliability at time t, where $t$ is continuous. Simply when cycle times are deterministically known $\mathrm{R}(\mathrm{t})$, , where is instant time at which the ith cycle occurs. The time dependent load was discussed by several researchers. M.N.Gopalan studied reliability analysis of time dependent cascade system with deterministic cycle times.

In the present paper has been carried out to find the reliability for time dependent stress-strength system using finite mixture of exponential distribution and also estimation of stress- strength reliability.

\section{Statistical Model:-}

$\mathrm{X}$ and $\mathrm{Y}$ denote the stress and strength of the system. $\mathrm{f}(\mathrm{X})$ and $\mathrm{g}(\mathrm{Y})$ are probability density functions of $\mathrm{X}$ and $\mathrm{Y}$.

Then the reliability of the system is

$$
R=\int_{0}^{\infty} f(x)\left(\int_{x}^{\infty} g(y) d y\right) d x
$$

For random independent stress and random- fixed strength the reliability of $\mathrm{n}$ cycles is

$$
R_{n}=\int_{0}^{\infty} g(y)\left(\int_{0}^{y} f(x) d x\right)^{n} d y
$$

If strength follows fimite mixture of exponential distribution and stress follows exponential distribution, i.e.

$$
\begin{gathered}
g_{i}(y)=\mu_{i} \exp \left(-\mu_{i} y\right), \quad \mu_{i}>0, y>0 \\
f(x)=\lambda \exp (-\lambda x), \lambda>0, x>0
\end{gathered}
$$

For $\mathrm{k}=1$, i.e. strength is mixture of one component

$$
\begin{gathered}
R_{n}=\int_{0}^{\infty} \mu_{1} e^{-\mu_{1} y}\left(\int_{0}^{y} \lambda e^{-\lambda x} d x\right)^{n} d y \\
R_{n}=\int_{0}^{\infty} \mu_{1} e^{-\mu_{1} y}\left(1-e^{-\lambda y}\right)^{n} d y \\
R_{n}=\frac{\lambda^{n} n(n-1)(n-2) \ldots \ldots .}{\left(\mu_{1}+\lambda\right)\left(\mu_{1}+2 \lambda\right)\left(\mu_{1}+3 \lambda\right) \ldots \ldots . .} \\
R_{n}=\frac{n ! \lambda^{n}}{\left(\mu_{1}+\lambda\right)\left(\mu_{1}+2 \lambda\right)\left(\mu_{1}+3 \lambda\right) \ldots \ldots}
\end{gathered}
$$

For $\mathrm{k}=2$, i.e. strength is mixture of two components

$$
\begin{gathered}
R_{n}=\int_{0}^{\infty}\left(\mu_{1} e^{-\mu_{1} y}+\mu_{2} e^{-\mu_{2} y}\right)\left(\int_{0}^{y} \lambda e^{-\lambda x} d x\right)^{n} d y \\
R_{n}=\int_{0}^{\infty}\left(\mu_{1} e^{-\mu_{1} y}+\mu_{2} e^{-\mu_{2} y}\right)\left(1-e^{-\lambda y}\right)^{n} d y \\
R_{n}=\int_{0}^{\infty}\left[\left(\mu_{1} e^{-\mu_{1} y}\left(1-e^{-\lambda y}\right)^{n}+\mu_{2} e^{-\mu_{2} y}\left(1-e^{-\lambda y}\right)^{n}\right)\right] d y \\
R_{n}=\lambda^{n} n(n-1)(n-2) \ldots \ldots\left[\frac{1}{\left(\mu_{1}+\lambda\right)\left(\mu_{1}+2 \lambda\right)\left(\mu_{1}+3 \lambda\right) \ldots \ldots\left(\mu_{1}+(n-1) \lambda\right)}\right. \\
\left.+\frac{1}{\left(\mu_{2}+\lambda\right)\left(\mu_{2}+2 \lambda\right)\left(\mu_{2}+3 \lambda\right) \ldots \ldots\left(\mu_{2}+(n-1) \lambda\right)}\right]
\end{gathered}
$$




$$
\begin{aligned}
& R_{n}=n ! \lambda^{n}\left[\frac{1}{\left(\mu_{1}+\lambda\right)\left(\mu_{1}+2 \lambda\right)\left(\mu_{1}+3 \lambda\right) \ldots \ldots\left(\mu_{1}+(n-1) \lambda\right)}\right. \\
&\left.+\frac{1}{\left(\mu_{2}+\lambda\right)\left(\mu_{2}+2 \lambda\right)\left(\mu_{2}+3 \lambda\right) \ldots \ldots\left(\mu_{2}+(n-1) \lambda\right)}\right]
\end{aligned}
$$

For $\mathrm{k}=3$, i.e. strength is mixture of three components

$$
\begin{array}{r}
R_{n}=\int_{0}^{\infty}\left(\mu_{1} e^{-\mu_{1} y}+\mu_{2} e^{-\mu_{2} y}+\mu_{3} e^{-\mu_{3} y}\right)\left(\int_{0}^{y} \lambda e^{-\lambda x} d x\right)^{n} d y \\
R_{n}=\int_{0}^{\infty}\left(\mu_{1} e^{-\mu_{1} y}+\mu_{2} e^{-\mu_{2} y}+\mu_{3} e^{-\mu_{3} y}\right)\left(1-e^{-\lambda y}\right)^{n} d y \\
R_{n}=n ! \lambda^{n}\left[\frac{1}{\left(\mu_{1}+\lambda\right)\left(\mu_{1}+2 \lambda\right)\left(\mu_{1}+3 \lambda\right) \ldots \ldots\left(\mu_{1}+(n-1) \lambda\right)}\right. \\
\left.\quad+\frac{1}{\left(\mu_{2}+\lambda\right)\left(\mu_{2}+2 \lambda\right)\left(\mu_{2}+3 \lambda\right) \ldots \ldots\left(\mu_{2}+(n-1) \lambda\right)}\right] \\
\left.\quad+\frac{1}{\left(\mu_{3}+\lambda\right)\left(\mu_{2}+2 \lambda\right)\left(\mu_{2}+3 \lambda\right) \ldots \ldots\left(\mu_{3}+(n-1) \lambda\right)}\right]
\end{array}
$$

In general

$$
R_{n}=n ! \lambda^{n} \sum_{k=1}^{n} \frac{1}{\left(\mu_{k}+\lambda\right)\left(\mu_{k}+2 \lambda\right)\left(\mu_{k}+3 \lambda\right) \ldots \ldots\left(\mu_{k}+(n-1) \lambda\right)}
$$

\section{Conclusion:-}

In this paper to find the reliability of finite mixture model where strength follow finite mixture of exponential distribution and stress follows exponential distribution. The calculations are done for specific values of stress strength partameters. It can interpret that stress parameter increases, the reliability decreases and strength parameter increases, reliability also increases

\section{References:-}

1. Bilikam, J.Edward(1985) : Some stochastic Stress- Strength processes, vol .R-34, pp: 269-274.

2. Kecheng Shen(1988): On the relation between component failure rate and stree - strength distributional charecterstics, Micro Electronics Reliability, vol. 28 , pp:801-812.

3. M.N.Gopalan and P.Venkateswarlu(1982) : reliability analysis of time dependent cascade system with deterministic cycle times, Micro Electronics Reliability, vol. 22, pp:841-872.

4. Dong Shang Chang (1995) : Reliability bounds for the stress-strength model, vol.29, pp:15-19.

5. Kapur,K.C. and L.R.Lamberson(1977) : Reliability in Engineering Design, Jhon Wiley and

6. sons, Inc., New York.

7. S.C.Gupta and V.K.Kapoor : Fundamentals of Mathematical Statistics.

8. M.N.Gopalan and Venkateswarlu (1983) : Reliability analysis of time dependent cascade system with random cycle times, vol. 23, pp:355-366.

9. T.S.Uma Maheswari (1993) : Reliability comparison of an $\mathrm{n}$ - cascade system with the addition of an nstrength system, Micro Electron Reliability, Vol. 33, No. 4, pp: 477-479, Pergamon Press, OXPORD. 